\title{
On the Approximate Controllability for Higher Order Parabolic Nonlinear Equations of Cahn-Hilliard Type
}

\author{
J.I. Díaz \\ Dpto. Matemática Aplicada \\ Universidad Complutense de Madrid \\ Avda. Complutense s/n, 28040, Madrid
}

and

\author{
A.M. Ramos \\ Dpto. Informática y Automática \\ Universidad Complutense de Madrid \\ Avda. Complutense s/n, 28040, Madrid.
}

\begin{abstract}
We prove the approximate controllability property for some higher order parabolic nonlinear equations of Cahn-Hilliard type when the nonlinearity is of sublinear type at infinity. We also give a counterexample showing that this property may fail when the nonlinearity is of superlinear type.
\end{abstract}

1991 Mathematical Subject Classification. 93B05, 93C20, 35K55.

Keywords. Approximate Controllability, Higher order nonlinear parabolic boundary value problems, Cahn-Hilliard type equations.

\section{Introduction.}

Let $\Omega$ be a bounded open subset of $\mathbb{R}^{N}$ of class $C^{2 m}, T>0, \omega$ a nonempty open subset of $\Omega, f$ a continuous real function and $k \in \mathbb{N}$ such that $0 \leq 2 k \leq m$. The main goal of this work is the study of the approximate controllability of the following semilinear equation with Dirichlet boundary conditions:

$$
\begin{cases}y_{t}+(-\Delta)^{m} y+(-\Delta)^{k} f(y)=h+v \chi_{\omega} & \text { in } Q:=\Omega \times(0, T), \\ \frac{\partial^{j} y}{\partial \nu^{j}}=0 \quad, \quad j=0,1, \cdots, m-1 & \text { on } \Sigma:=\partial \Omega \times(0, T), \\ y(0)=y_{0} & \text { in } \Omega,\end{cases}
$$

where $v$ is a suitable output control, $\chi_{\omega}$ is the characteristic function of $\omega, \nu$ is the unit outward normal vector, $h \in L^{2}\left(0, T: H^{-m}(\Omega)\right)$ and $y_{0} \in L^{2}(\Omega)$. Due to the term $\chi_{\omega}$ the controls are assumed supported on the set $\mathcal{O}:=\omega \times(0, T)$. Problems as (1), sometimes known as Cahn-Hilliard problems, appear, with $m=2$, in the study of phase separation in cooling binary solutions and in other contexts generating spatial pattern formation (see [6], [8] and the references cited therein).

We recall that problem (1) satisfies the approximate controllability property, at time $T$ with states space $X$ and controls space $Y$, if the set

$$
\{y(T, \cdot: v): v \in Y, y \text { solution of }(1)\}
$$

is dense in $X$.

The main goal of this paper is to extend the approximate controllability results on second order problems, $m=1$ and $k=0$ (see e.g. [9], [10] and [7]) to the case of higher order equations for which the maximum principle does not hold, in general. Our first result gives a positive answer when $f$ is assumed to be sublinear at the infinity:

Theorem 1 Assume that $f$ satisfies the following conditions: there exist some positive constants $c_{1}$ and $c_{2}$ such that

$$
|f(s)| \leq c_{1}+c_{2}|s| \quad \text { for all } s \in \mathbb{R}
$$

and

$$
\text { there exists } f^{\prime}\left(s_{0}\right) \text { for some } s_{0} \in \mathbb{R} \text {. }
$$

Then problem (1) satisfies the approximate controllability property at time $T$ with states space $X=L^{2}(\Omega)$ and controls space $Y=L^{2}(\mathcal{O})$. 
In contrast to the above result, we shall prove that when $f$ is superlinear the approximate controllability property does not hold in general, as explained in Section 4. Therefore if, for instance, $f(s)=|s|^{p-1} s$ Theorem 1 gives a positive approximate controllability result for $0<p \leq 1$. The results of section 6 provide a negative approximate controllability answer when $1<p<\infty$. The similar alternative was obtained in Díaz-Ramos [7] for second order parabolic semilinear problems.

We remark that the existence of solutions in the class

$$
y \in L^{2}\left(0, T ; H_{0}^{m}(\Omega)\right) \cap C\left([0, T] ; L^{2}(\Omega)\right), f(y) \in L^{2}(Q), \Delta^{k} f(y) \in L^{2}\left(0, T ; H^{-m}(\Omega)\right),
$$

is also obtained as a by-product of Theorem 1 for a suitable subclass of controls. The uniqueness of solutions can be easily proved if, for instance, $f$ is nondecreasing or Lipschitz continuous. Those uniqueness results are not needed in our arguments.

\section{Approximate Controllability for an Associated Linear problem.}

In order to prove Theorem 1 we follows the same scheme of proof than in [9], [10] and [7]. We define the function

$$
g(s)=\frac{f(s)-f\left(s_{0}\right)}{s-s_{0}} .
$$

From assumptions (2) and (3) we have that $g \in L^{\infty}(\mathbb{R}) \cap \mathcal{C}(\mathbb{R})$. The conclusion will be derived from a fixed point argument. As $f(s)=f\left(s_{0}\right)+g(s) s-g(s) s_{0}$, we shall start by considering the approximate controllability for a linear problem obtained by replacing the term $f(y)$ by

$$
g(z) y+f\left(s_{0}\right)-g(z) s_{0},
$$

where $z$ is an arbitrary function in $L^{2}(Q)$. Notice that when $z=y$ this expression coincides with $f(y)$ and that if we denote $g(z(t, x)):=a(t, x)$ and

$$
h(a):=-(-\Delta)^{k} f\left(s_{0}\right)+(-\Delta)^{k}\left(a(t, x) s_{0}\right),
$$

then $a \in L^{\infty}(Q)$ and $h(a) \in L^{\infty}\left(0, T ; H^{-2 k}(\Omega)\right)$. More in general, given $a \in L^{\infty}(Q)$ and $h(a)$ defined by (4), we consider the approximate controllability property corresponding to the linear problem

$$
\begin{cases}y_{t}+(-\Delta)^{m} y+(-\Delta)^{k}(a(t, x) y)=h+h(a)+u \chi_{\omega} & \text { in } Q:=\Omega \times(0, T), \\ \frac{\partial^{j} y}{\partial \nu^{j}}=0 \quad, \quad j=0,1, \cdots, m-1 & \text { on } \Sigma:=\partial \Omega \times(0, T), \\ y(0)=y_{0} & \text { in } \Omega .\end{cases}
$$

Before stating an approximate controllability result for this problem, following Lions [14] and Fabre-PuelZuazua [9], [10], we consider $\varepsilon>0$ and $y_{d} \in L^{2}(\Omega)$ and we introduce the functional $J=J\left(\cdot ; a, y_{d}\right): L^{2}(\Omega) \rightarrow$ $\mathbb{R}$ defined by

$$
J\left(\varphi_{0} ; a, y_{d}\right)=J\left(\varphi^{0}\right)=\frac{1}{2}\left(\int_{\mathcal{O}}|\varphi(t, x)| d x d t\right)^{2}+\varepsilon\left\|\varphi^{0}\right\|_{L^{2}(\Omega)}-\int_{\Omega} y_{d} \varphi^{0}, d x
$$

where $\varphi(t, x)$ is the solution of the backward problem

$$
\begin{cases}-\varphi_{t}+(-\Delta)^{m} \varphi+a(t, x) \Delta^{k} \varphi=0 & \text { in } Q:=\Omega \times(0, T), \\ \frac{\partial^{j} \varphi}{\partial \nu^{j}}=0, \quad j=0,1, \cdots, m-1 & \text { on } \Sigma:=\partial \Omega \times(0, T), \\ \varphi(T)=\varphi^{0} & \text { in } \Omega .\end{cases}
$$

To study the above backward problem we introduce the space

$$
W:=\left\{y \in L^{2}\left(0, T ; H_{0}^{m}(\Omega)\right), y_{t} \in L^{2}\left(0, T ; H^{-m}(\Omega)\right)\right\} .
$$

The following result will be used later 
Proposition 1 Given $h \in L^{2}\left(0, T ; H^{-m}(\Omega)\right)$ and $y_{0} \in L^{2}(\Omega)$, there exists a unique function $y \in W$ satisfying

$$
\begin{cases}y_{t}+(-\Delta)^{m} y+a(t, x) \Delta^{k} y=h & \text { in } Q \\ \frac{\partial^{j} y}{\partial \nu^{j}}=0 \quad, \quad j=0,1, \cdots, m-1 & \text { on } \Sigma \\ y(0)=y_{0} & \text { in } \Omega\end{cases}
$$

Furthermore, we have the estimate

$$
\|y\|_{L^{2}\left(0, T ; H_{0}^{m}(\Omega)\right)}+\left\|y_{t}\right\|_{L^{2}\left(0, T ; H^{-m}(\Omega)\right)} \leq C\left(\|h\|_{L^{2}\left(0, T ; H^{-m}(\Omega)\right)}+\left\|y_{0}\right\|_{L^{2}(\Omega)}\right),
$$

where the constant $C$ depends only on $M:=\|a\|_{L^{\infty}(Q)}$ (provided that $\Omega, T$ and $m$ are kept fixed). Moreover, if $h \in L^{2}(Q)$, the solution $y$ also satisfies that

$$
y \in L^{2}\left(\delta, T ; H^{2 m}(\Omega)\right) \quad \text { and } \quad y_{t} \in L^{2}((\delta, T) \times \Omega) \quad \text { for all } \delta \in(0, T) .
$$

Proof. For all $n \in \mathbb{N}$ we define $y^{n+1}$ as the solution of the following iterative problem

$$
\begin{cases}y_{t}^{n+1}+(-\Delta)^{m} y^{n+1}=h-a(t, x) \Delta^{k} y^{n} & \text { in } Q \\ \frac{\partial^{j} y^{n+1}}{\partial \nu^{j}}=0 \quad, \quad j=0,1, \cdots, m-1 & \text { on } \Sigma \\ y^{n+1}(0)=y_{0} & \text { in } \Omega\end{cases}
$$

where $y^{0}(t):=0$ for all $t \in[0, T]$. The existence of a solution $y^{n} \in W$ can be found, for instance, in Theorem 3.4.1 of Lions-Magenes [15]. Thus, for all $n \in \mathbb{N} \backslash\{0,1\}, y^{n+1}-y^{n}$ satisfies

$$
\begin{cases}\left(y^{n+1}-y^{n}\right)_{t}+(-\Delta)^{m}\left(y^{n+1}-y^{n}\right)=-a(t, x) \Delta^{k}\left(y^{n}-y^{n-1}\right) & \text { in } Q \\ \frac{\partial^{j}\left(y^{n+1}-y^{n}\right)}{\partial \nu^{j}}=0 \quad, \quad j=0,1, \cdots, m-1 & \text { on } \Sigma \\ \left(y^{n+1}-y^{n}\right)(0)=0 & \text { in } \Omega\end{cases}
$$

and therefore

$$
y^{n+1}-y^{n} \in H^{1,2 m}(Q):=H^{1}\left(0, T ; L^{2}(\Omega)\right) \cap L^{2}\left(0, T ; H^{2 m}(\Omega)\right)
$$

and

$$
\left\|y^{n+1}-y^{n}\right\|_{H^{1,2 m}(Q)} \leq c_{1}\left\|a \Delta^{k}\left(y^{n}-y^{n-1}\right)\right\|_{L^{2}(Q)}
$$

(see, for instance, Theorem 4.6.1 of Lions-Magenes [16]). Then, since

$$
H^{1,2 m}(Q) \subset \mathcal{C}\left([0, T] ; H^{m}(\Omega)\right)
$$

with continuous imbedding (see, for instance, Theorems 1.3.1 and 1.9.6 of Lions-Magenes [15]), there exists $c_{2}=c_{2}(T)$ such that

$$
\left\|y^{n+1}-y^{n}\right\|_{\mathcal{C}\left([0, T] ; H_{0}^{m}(\Omega)\right)} \leq c_{2}\left\|a \Delta^{k}\left(y^{n}-y^{n-1}\right)\right\|_{L^{2}(Q)} .
$$

Further, it is clear that we can choose $C_{2}=C_{2}(T)$ such that for all $t \in[0, T]$

$$
\left\|y^{n+1}-y^{n}\right\|_{\mathcal{C}\left([0, t] ; H_{0}^{m}(\Omega)\right)} \leq C_{2}\left\|a \Delta^{k}\left(y^{n}-y^{n-1}\right)\right\|_{L^{2}((0, t) \times \Omega)} .
$$

Hence,

$$
\left\|\left(y^{n+1}-y^{n}\right)(t)\right\|_{H_{0}^{m}(\Omega)}^{2} \leq\left(C_{2} M\right)^{2} \int_{0}^{t}\left\|\Delta^{k}\left(y^{n}-y^{n-1}\right)(\tau)\right\|_{L^{2}(\Omega)}^{2} d \tau, \quad \text { for all } t \in[0, T]
$$

and therefore, by using the Poincaré inequality, there exists a constant $K$, independent of $M$, such that

$$
\left\|\left(y^{n+1}-y^{n}\right)(t)\right\|_{H_{0}^{m}(\Omega)}^{2} \leq(K M)^{2} \int_{0}^{t}\left\|\left(y^{n}-y^{n-1}\right)(\tau)\right\|_{H_{0}^{m}(\Omega)}^{2} d \tau, \quad \text { for all } t \in[0, T] .
$$

Then, for every $t \in[0, T]$ we deduce that

$$
\left\|\left(y^{n+1}-y^{n}\right)(t)\right\|_{H_{0}^{m}(\Omega)}^{2} \leq\left(K^{2} M^{2}\right)^{n-1} \int_{0}^{t} \int_{0}^{\tau_{1}} \cdots \int_{0}^{\tau_{n-1}}\left\|\left(y^{2}-y^{1}\right)\left(\tau_{n}\right)\right\|_{H_{0}^{m}(\Omega)}^{2} d \tau_{n} \cdots d \tau_{1}
$$




$$
\begin{gathered}
\leq\left(K^{2} M^{2}\right)^{n-1} \int_{0}^{t} \int_{0}^{\tau_{1}} \cdots \int_{0}^{\tau_{n-1}}\left\|y^{2}-y^{1}\right\|_{\mathcal{C}\left([0, T] ; H_{0}^{m}(\Omega)\right)}^{2} d \tau_{n} \cdots d \tau_{1} \\
\leq\left(K^{2} M^{2}\right)^{n-1} \frac{t^{n-1}}{(n-1) !}\left\|y^{2}-y^{1}\right\|_{\mathcal{C}\left([0, T] ; H_{0}^{m}(\Omega)\right)}^{2} \\
\leq \frac{\left(K^{2} M^{2} T\right)^{n-1}}{(n-1) !}\left\|y^{2}-y^{1}\right\|_{\mathcal{C}\left([0, T] ; H_{0}^{m}(\Omega)\right)}^{2},
\end{gathered}
$$

which implies that

$$
\left\|y^{n+1}-y^{n}\right\|_{\mathcal{C}\left([0, T] ; H_{0}^{m}(\Omega)\right)} \rightarrow 0 \quad \text { as } n \rightarrow \infty
$$

and therefore, by (11), we deduce that

$$
\left\|\left(y^{n+1}-y^{n}\right)_{t}\right\|_{L^{2}\left(0, T ; H^{-m}(\Omega)\right)} \rightarrow 0 \quad \text { as } n \rightarrow \infty .
$$

Then, there exists $y \in W$ such that

$$
y_{n} \rightarrow y \quad \text { in } W \text { as } n \rightarrow \infty .
$$

In order to prove that $y$ satisfies (8) we point out that

$$
\begin{gathered}
\Delta^{m} y^{n} \rightarrow \Delta^{m} y \quad \text { in } L^{2}\left(0, T ; H^{-m}(\Omega)\right) \text { as } n \rightarrow \infty, \\
\Delta^{k} y^{n} \rightarrow \Delta^{k} y \quad \text { in } L^{2}(\Omega) \text { as } n \rightarrow \infty,
\end{gathered}
$$

and

$$
y_{t}^{n} \rightarrow y_{t} \quad \text { in } L^{2}\left(0, T ; H^{-m}(\Omega)\right) \text { as } n \rightarrow \infty .
$$

this implies (passing to the limit) that $y$ is the solution of (8). In order to prove (9), we "multiply" in (8) by $y$. Then it is easy to see that

$$
\|y\|_{L^{2}\left(0, T ; H_{0}^{m}(\Omega)\right)}+\left\|y_{t}\right\|_{L^{2}\left(0, T ; H^{-m}(\Omega)\right)} \leq C\left(\|h\|_{L^{2}\left(0, T ; H^{-m}(\Omega)\right)}+\left\|y_{0}\right\|_{L^{2}(\Omega)}+\|y\|_{L^{2}(Q)}\right) .
$$

Furthermore,

$$
\|y(t)\|_{L^{2}(\Omega)}^{2} \leq\left(\|y(0)\|_{L^{2}(\Omega)}^{2}+c_{2}\|h\|_{L^{2}\left(0, T ; H^{-m}(\Omega)\right)}^{2}\right)+c_{3} \int_{0}^{t}\|y(s)\|_{L^{2}(\Omega)}^{2} d s .
$$

Then, applying Gronwall's inequality (see, for instance, Lemma 4 of Haraux [11]), we deduce that

$$
\|y(t)\|_{L^{2}(\Omega)}^{2} \leq\left(\|y(0)\|_{L^{2}(\Omega)}^{2}+c_{2}\|h\|_{L^{2}\left(0, T ; H^{-m}(\Omega)\right)}^{2}\right) e^{c_{3} t} \quad \forall t \in[0, T] .
$$

From here, we obtain that

$$
\|y\|_{L^{2}(Q)} \leq c_{4}\left(\|h\|_{L^{2}\left(0, T ; H^{-m}(\Omega)\right)}+\left\|y_{0}\right\|_{L^{2}(\Omega)}\right)
$$

which implies, together with (12), inequality (9). Now, thanks to (9) and the linearity of Problem (8), we deduce the uniqueness of solution.

Finally, if $h \in L^{2}(Q)$, since $y(\delta) \in H_{0}^{m}(\Omega)$ for all $\delta \in(0, T)$, taking $y(\delta)$ as initial datum and applying Theorem 4.6 .1 of [16], we get (10).

As usual in Controllability Theory we shall use a unique continuation property for solutions of the dual problem (in our case Problem (7)).

Lemma 1 Let $\omega$ be a nonempty open subset of $\Omega$. Assume that

$$
\varphi \in L^{2}\left(0, T ; H_{0}^{m}(\Omega)\right) \cap C\left([0, T] ; L^{2}(\Omega)\right)
$$

satisfies (7) and that $\varphi \equiv 0$ in $\mathcal{O}=\omega \times(0, T)$. Then $\varphi \equiv 0$ in $Q$.

Proof. From Proposition 1 (applied with backward time) we deduce that $\varphi \in L^{2}\left(0, T-\delta ; H^{2 m}(\Omega)\right)$ for all $\delta \in(0, T)$. Then Lemma 1 follows from Theorem 3.2 of Saut-Scheurer [17].

The following two results are easy adaptations (by using Lemma 1) of the similar ones given in [9], [10] for second order parabolic problems. 
Proposition 2 The functional $J\left(\cdot ; a, y_{d}\right)$ is continuous, strictly convex on $L^{2}(\Omega)$ and verifies

$$
\liminf _{\left\|\varphi^{0}\right\|_{L^{2}(\Omega)} \rightarrow \infty} \frac{J\left(\varphi^{0} ; a, y_{d}\right)}{\left\|\varphi^{0}\right\|_{L^{2}(\Omega)}} \geq \varepsilon .
$$

Further $J\left(\cdot ; a, y_{d}\right)$ attains its minimum at a unique point $\widehat{\varphi}^{0}$ in $L^{2}(\Omega)$ and

$$
\widehat{\varphi}^{0}=0 \quad \Leftrightarrow \quad\left\|y_{d}\right\|_{L^{2}(\Omega)} \leq \varepsilon .
$$

Proposition 3 Let $\mathcal{M}$ be the mapping

$$
\begin{array}{cccc}
\mathcal{M}: \quad L^{\infty}(Q) \times L^{2}(\Omega) & \rightarrow & L^{2}(\Omega) \\
\left(a(t, x), y_{d}\right) & \longrightarrow & \widehat{\varphi}^{0} .
\end{array}
$$

If $B$ is a bounded subset of $L^{\infty}(Q)$ and $K$ is a compact subset of $L^{2}(\Omega)$, then $\mathcal{M}(B \times K)$ is a bounded subset of $L^{2}(\Omega)$.

In order to characterize the duality of problem (7), we recall that given a convex and proper function $V: X \rightarrow \mathbb{R} \cup\{+\infty\}$ on the Banach space $X$, it is said that a element $p_{0}$ of $V^{\prime}$ belongs to the set $\partial V\left(x_{0}\right)$ (subdifferential of $V$ at $x_{0} \in X$ ) if

$$
V\left(x_{0}\right)-V(x) \leq\left(p_{0}, x_{0}-x\right) \quad \forall x \in X .
$$

It is well known that that if $V$ is Gateaux differentiable its differential coincides with its subdifferential and that $x_{0}$ minimizes $V$ over $X$ (or over a convex subset of $X$ ) if and only if $0 \in \partial V\left(x_{0}\right)$. Finally, if $V$ is a lower semicontinuous function, then $p_{0} \in \partial V\left(x_{0}\right)$ if and only if

$$
\left(p_{0}, x\right) \leq \lim _{h \rightarrow 0^{+}} \frac{V\left(x_{0}+h x\right)-V\left(x_{0}\right)}{h}(<+\infty) \quad \forall x \in X .
$$

(See, for instance, Aubin-Ekeland [3]). Coming back to the functional $J$ we have:

Lemma 2 For every $\varphi^{0} \in L^{2}(\Omega)\left(\varphi^{0} \neq 0\right)$, if $\varphi$ is the solution of $(7)$ satisfying $\varphi(T)=\varphi^{0}$, we have that

$$
\begin{aligned}
& \partial J\left(\varphi^{0} ; a, y_{d}\right)=\left\{\xi \in L^{2}(\Omega), \exists v \in \operatorname{sgn}(\varphi) \chi_{\mathcal{O}}\right. \text { satisfying } \\
& \int_{\Omega} \xi(x) \theta^{0}(x) d x=\left(\int_{\mathcal{O}}|\varphi(t, x)| d \Sigma\right)\left(\int_{\mathcal{O}} v(t, x) \theta(t, x) d \Sigma\right) \\
&\left.+\varepsilon \int_{\Omega} \frac{\varphi^{0}(x)}{\left\|\varphi^{0}\right\|_{L^{2}(\Omega)}} \theta^{0}(x) d x-\int_{\Omega} y_{d}(x) \theta^{0}(x) d x \forall \theta^{0} \in L^{2}(\Omega)\right\}
\end{aligned}
$$

where $\theta$ is the solution of (7) satisfying $\theta(T)=\theta^{0}$.

Proof. It is an easy modification of Proposition 2.4 of [10].

Let us prove the approximate controllability property for an special version of the linear problem given in $(5)$.

Theorem 2 If $\left\|y_{d}\right\|_{L^{2}(\Omega)}>\varepsilon$ and $\widehat{\varphi}$ is the solution of $(7)$ corresponding to $\widehat{\varphi}(T)=\widehat{\varphi}^{0}$, with $\widehat{\varphi}^{0}$ minimum of $J\left(\cdot ; a, y_{d}\right)$. Then there exists $v \in \operatorname{sgn}(\widehat{\varphi}) \chi_{\mathcal{O}}$ such that the solution of

$$
\begin{cases}y_{t}+(-\Delta)^{m} y+\left(-\Delta^{k}\right)(a(t, x) y)=\|\widehat{\varphi}\|_{L^{1}(\mathcal{O})} v \chi_{\mathcal{O}} & \text { in } Q, \\ \frac{\partial^{j} y}{\partial \nu^{j}}=0 \quad(j=0 \cdots(m-1)) & \text { on } \Sigma, \\ y(0)=0 & \text { in } \Omega,\end{cases}
$$

satisfies

$$
y(T)=y_{d}-\varepsilon \frac{\widehat{\varphi}^{0}}{\left\|\widehat{\varphi}^{0}\right\|_{L^{2}(\Omega)}},
$$

and then $\left\|y(T)-y_{d}\right\|_{L^{2}(\Omega)}=\varepsilon$. 
Remark 1 In the case $\left\|y_{d}\right\|_{L^{2}(\Omega)} \leq \varepsilon$, if we use the null control, we obtain $y=0$ and therefore $\| y(T)-$ $y_{d} \|_{L^{2}(\Omega)} \leq \varepsilon$.

First of all we prove the existence and uniqueness to problem given by (5).

Proposition 4 Assumed $y_{0} \in L^{2}(\Omega), h \in L^{2}\left(0, T ; H^{-m}(\Omega)\right)$ and $a(t, x) \in L^{\infty}(Q)$, there exists a unique function $y \in W$ satisfying

$$
\begin{cases}y_{t}+(-\Delta)^{m} y+\Delta^{k}(a(t, x) y)=h & \text { in } Q \\ \frac{\partial^{j} y}{\partial \nu^{j}}=0 \quad, \quad j=0,1, \cdots, m-1 & \text { on } \Sigma, \\ y(0)=y_{0} & \text { in } \Omega .\end{cases}
$$

Moreover, we have the estimate

$$
\|y\|_{L^{2}\left(0, T ; H_{0}^{m}(\Omega)\right)}+\left\|y_{t}\right\|_{L^{2}\left(0, T ; H^{-m}(\Omega)\right.} \leq C\left(\|h\|_{L^{2}\left(0, T ; H^{-m}(\Omega)\right)}+\left\|y_{0}\right\|_{L^{2}(\Omega)}\right),
$$

where the constant $C$ depends only on $M$ (provided that $\Omega, T$ and $m$ are kept fixed).

Proof. For all $n \in \mathbb{N}$ we define again $y^{n+1}$ as the solution of the iterative problem

$$
\begin{cases}y_{t}^{n+1}+(-\Delta)^{m} y^{n+1}=h-\Delta^{k}\left(a(t, x) y^{n}\right) & \text { in } Q \\ \frac{\partial^{j} y^{n+1}}{\partial \nu^{j}}=0 \quad, \quad j=0,1, \cdots, m-1 & \text { on } \Sigma \\ y^{n+1}(0)=y_{0} & \text { in } \Omega\end{cases}
$$

where $y^{0}(t):=0$ for all $t \in[0, T]$. The existence of a solution $y^{n} \in W$ can be found, for instance, in Theorem 3.4.1 of Lions-Magenes [15]. Thus, for all $n \in \mathbb{N} \backslash\{0,1\}, y^{n+1}-y^{n}$ is solution of

$$
\begin{cases}\left(y^{n+1}-y^{n}\right)_{t}+(-\Delta)^{m}\left(y^{n+1}-y^{n}\right)=-\Delta^{k}\left[a(t, x)\left(y^{n}-y^{n-1}\right)\right] & \text { in } Q, \\ \frac{\partial^{j}\left(y^{n+1}-y^{n}\right)}{\partial \nu^{j}}=0 \quad, \quad j=0,1, \cdots, m-1 & \text { on } \Sigma, \\ \left(y^{n+1}-y^{n}\right)(0)=0 & \text { in } \Omega\end{cases}
$$

and therefore (see again Theorem 3.4.1 of Lions-Magenes [15]) $y^{n+1}-y^{n} \in W$ and

$$
\left\|y^{n+1}-y^{n}\right\|_{W} \leq c_{1}\left\|a\left(y^{n}-y^{n-1}\right)\right\|_{L^{2}(Q)} .
$$

Then, since $W \subset \mathcal{C}\left([0, T] ; L^{2}(\Omega)\right.$ ) with continuous imbedding (see, for instance, [12] or [15]), we have that

$$
\left\|y^{n+1}-y^{n}\right\|_{\mathcal{C}\left([0, T] ; L^{2}(\Omega)\right)} \leq c_{2}\left\|a\left(y^{n}-y^{n-1}\right)\right\|_{L^{2}(Q)} .
$$

Further, as in the proof of Proposition 1, we can choose $C_{2}=C_{2}(T)$ such that

$$
\left\|y^{n+1}-y^{n}\right\|_{\mathcal{C}\left([0, t] ; L^{2}(\Omega)\right)} \leq C_{2}\left\|a\left(y^{n}-y^{n-1}\right)\right\|_{L^{2}((0, t) \times \Omega)}, \quad \text { for all } t \in[0, T] .
$$

Hence,

$$
\left\|\left(y^{n+1}-y^{n}\right)(t)\right\|_{L^{2}(\Omega)}^{2} \leq\left(C_{2} M\right)^{2} \int_{0}^{t}\left\|\left(y^{n}-y^{n-1}\right)(\tau)\right\|_{L^{2}(\Omega)}^{2} d \tau, \quad \text { for all } t \in[0, T]
$$

Then, for every $t \in[0, T]$ we deduce that

$$
\begin{gathered}
\left\|\left(y^{n+1}-y^{n}\right)(t)\right\|_{L^{2}(\Omega)}^{2} \leq\left(C_{2}^{2} M^{2}\right)^{n-1} \int_{0}^{t} \int_{0}^{\tau_{1}} \cdots \int_{0}^{\tau_{n-1}}\left\|\left(y^{2}-y^{1}\right)\left(\tau_{n}\right)\right\|_{L^{2}(\Omega)}^{2} d \tau_{n} \cdots d \tau_{1} \\
\leq\left(C_{2}^{2} M^{2}\right)^{n-1} \int_{0}^{t} \int_{0}^{\tau_{1}} \cdots \int_{0}^{\tau_{n-1}}\left\|y^{2}-y^{1}\right\|_{\mathcal{C}\left([0, T] ; L^{2}(\Omega)\right)}^{2} d \tau_{n} \cdots d \tau_{1} \\
\leq\left(C_{2}^{2} M^{2}\right)^{n-1} \frac{t^{n-1}}{(n-1) !}\left\|y^{2}-y^{1}\right\|_{\mathcal{C}\left([0, T] ; L^{2}(\Omega)\right)}^{2} \\
\leq \frac{\left(C_{2}^{2} M^{2} T\right)^{n-1}}{(n-1) !}\left\|y^{2}-y^{1}\right\|_{\mathcal{C}\left([0, T] ; L^{2}(\Omega)\right)}^{2},
\end{gathered}
$$


which implies that

$$
\left\|y^{n+1}-y^{n}\right\|_{\mathcal{C}\left([0, T] ; L^{2}(\Omega)\right)} \rightarrow 0 \quad \text { as } n \rightarrow \infty
$$

and therefore, by (19), we deduce that

$$
\left\|y^{n+1}-y^{n}\right\|_{W} \rightarrow 0 \quad \text { as } n \rightarrow \infty .
$$

Then, there exists $y \in W$ such that

$$
y_{n} \rightarrow y \quad \text { in } W \text { as } n \rightarrow \infty .
$$

The end of the proof is similar to the end of the proof of Proposition 1.

Proof of Theorem 2. Using the subdifferentiability of $J\left(. ; a, y_{d}\right)$ at $\widehat{\varphi}^{0}(\neq 0$ by $(14))$, we know that

$$
0 \in \partial J\left(\widehat{\varphi}^{0}\right)
$$

which is equivalent, from Lemma 2 , to the existence of $v \in \operatorname{sgn}(\widehat{\varphi}) \chi_{\mathcal{O}}$, such that

$$
\begin{gathered}
-\|\widehat{\varphi}\|_{L^{1}(\mathcal{O})}\left(\int_{\mathcal{O}} v(x, t) \theta(x, t) d x d t\right)=\frac{\varepsilon}{\left\|\widehat{\varphi}^{0}\right\|_{L^{2}(\Omega)}} \int_{\Omega} \widehat{\varphi}^{0}(x) \theta^{0}(x) d x \\
-\int_{\Omega} y_{d}(x) \theta^{0}(x) d x .
\end{gathered}
$$

On the other hand, as $y \in W$, if we "multiply" by $\theta$ in (15) we obtain, by (7), that

$$
\int_{\Omega} y(T, x) \theta^{0}(x) d x d t=\|\widehat{\varphi}\|_{L^{1}(\mathcal{O})}\left(\int_{\mathcal{O}} v(x, t) \theta(x, t) d x d t\right)
$$

Then, from (20) and (21), we obtain

$$
\int_{\Omega} y(T, x) \theta^{0}(x) d x d t=\int_{\Omega}\left(y_{d}(x)-\varepsilon \frac{\widehat{\varphi}^{0}(x)}{\left\|\widehat{\varphi}^{0}\right\|_{L^{2}(\Omega)}}\right) \theta^{0}(x) d x d t \quad \forall \theta^{0} \in L^{2}(\Omega)
$$

and we conclude that $y(T)=y_{d}-\varepsilon \frac{\widehat{\varphi}^{0}}{\left\|\widehat{\varphi}^{0}\right\|_{L^{2}(\Omega)}}$.

Now we are ready to prove a linear version of Theorem 1 for problem (5)

Corollary 1 Let $\left\|y_{d}\right\|_{L^{2}(\Omega)}>\varepsilon$ and $\widehat{\varphi}$ the solution of (7) corresponding to $\widehat{\varphi}(T)=\widehat{\varphi}^{0}$, with $\widehat{\varphi}^{0}$ minimum of $J\left(\cdot ; a, y_{d}-y(T ; a, 0)\right)$, where in general $y(t ; a, u)$ denotes the solution of (5) corresponding to the control $u$. Then there exists $v \in \operatorname{sgn}(\widehat{\varphi}) \chi_{\mathcal{O}}$ such that the solution of

$$
\begin{cases}y_{t}+(-\Delta)^{m} y+\left(-\Delta^{k}\right)(a(t, x) y)=h+h(a)+\|\widehat{\varphi}\|_{L^{1}(\mathcal{O})} v \chi_{\mathcal{O}} & \text { in } Q, \\ \frac{\partial^{j} y}{\partial \nu^{j}}=0(j=0 \cdots(m-1)) & \text { on } \Sigma, \\ y(0)=y_{0} & \text { in } \Omega,\end{cases}
$$

satisfies

$$
\left\|y(T)-y_{d}\right\|_{L^{2}(\Omega)} \leq \varepsilon
$$

Proof. We put $y=L+Y$, where $L=L(a)$ satisfies

$$
\begin{cases}L_{t}+(-\Delta)^{m} L+\left(-\Delta^{k}\right)(a(t, x) L)=h+h(a) & \text { in } Q, \\ \frac{\partial^{j} L}{\partial \nu^{j}}=0(j=0 \cdots(m-1)) & \text { on } \Sigma, \\ L(0)=y_{0} & \text { in } \Omega\end{cases}
$$

and $Y=Y(a)$ is taken associated to the approximate controllability problem

$$
\begin{cases}Y_{t}+(-\Delta)^{m} Y+\left(-\Delta^{k}\right)(a(t, x) Y)=u(a) \chi_{\mathcal{O}} & \text { in } Q, \\ \frac{\partial^{j} Y}{\partial \nu^{j}}=0(j=0 \cdots(m-1)) & \text { on } \Sigma, \\ Y(0)=0 & \text { in } \Omega,\end{cases}
$$


with desired state $y_{d}-L(T)$, i.e. such that $\left\|Y(T)-\left(y_{d}-L(T)\right)\right\| \leq \varepsilon$. Notice that the existence of such a control $u(a)$ is consequence of Theorem 2. In particular, if $\left\|y_{d}-L(T)\right\| \leq \varepsilon$, we can take $u(a) \equiv 0$ and if $\left\|y_{d}-L(T)\right\|>\varepsilon$, then we take $u(a)=\|\widehat{\varphi}(a)\|_{L^{1}(\Omega)} v(a)$, where $v(a) \in \operatorname{sgn}(\widehat{\varphi}(a)) \chi_{\mathcal{O}}$ and $\widehat{\varphi}(a)$ is the solution of (7) with initial value $\mathcal{M}\left(\left(a(x, t), y_{d}-L(T)\right)\right)$ defined in Proposition 3. It is obvious that such function $y$ and such control $u(a)$ lead to the conclusion.

\section{Controllability for the nonlinear problem.}

As mentioned before, we shall use a fixed point argument to prove Theorem 1. In fact we shall deal with multivalued operators. Let us recall a well-known result: the Kakutani's fixed point Theorem. The usual continuity assumption in other fixed pont theorems is replaced here by the following notion:

Definition 1 Let $X, Y$ two Banach spaces and, $\Lambda: X \rightarrow \mathcal{P}(Y)$ a multivalued function. We say that $\Lambda$ is upper hemicontinuous at $x_{0} \in X$, if for every $p \in Y^{\prime}$, the function

$$
x \rightarrow \sigma(\Lambda(x), p)=\sup _{y \in \Lambda(x)}<p, y>_{Y^{\prime} \times Y}
$$

is upper semicontinuous at $x_{0}$. We say that the multivalued function is upper hemicontinuous on a subset $K$ of $X$, if it satisfies this properties for every point of $K$.

Theorem 3 (Kakutani's fixed point Theorem). Let $K \subset X$ be a convex and compact subset and $\Lambda: K \rightarrow K$ an upper hemicontinuous application with convex, closed and nonempty values. Then, there exists a fixed point $x_{0}$, of $\Lambda$.

For a proof see, for instance, Aubin [2].

Proof of Theorem 1. We fix $y_{d} \in L^{2}(\Omega)$ and $\varepsilon>0$. By using Corollary 1, for each $z \in L^{2}(Q)$ and $\varepsilon>0$ it is possible to find two functions $\varphi(z) \in L^{1}(Q)$ and $v(z) \in \operatorname{sgn}(\varphi(z)) \chi_{\mathcal{O}}$ such that the solution $y=y^{z}$ of

$$
\begin{cases}y_{t}+(-\Delta)^{m} y+(-\Delta)^{k}(g(z) y)=h+h(g(z))+u \chi_{\mathcal{O}} & \text { in } Q \\ \frac{\partial^{j} y}{\partial \nu^{j}}=0, j=0,1, \cdots m-1 & \text { on } \Sigma \\ y(0)=y_{0} & \text { in } \Omega\end{cases}
$$

(where $\left.u=u(z)=|\varphi(z)|_{L^{1}(\mathcal{O})} v(z)\right)$ satisfies

$$
\left|y(T)-y_{d}\right|_{L^{2}(\Omega)} \leq \varepsilon
$$

Here $\varphi(z)$ is the solution of (7) with initial value $M\left(\left(g(z), y_{d}-L(z ; T)\right)\right)$ (see Proposition 3$)$ and $a(t, x)=g(z)$, where is $L(z ; T)$ the solution of $(22)$, with $a=g(z)$, at time $T$.

Lemma 3 The set

$$
\left\{y_{d}-L(z ; T), z \in L^{2}(Q)\right\}
$$

is relatively compact in $L^{2}(\Omega)$.

Proof of Lemma 3. Applying Proposition 4 it is easy to see that the set of solutions $L(z)$ of

$$
\begin{cases}L_{t}+(-\Delta)^{m} L+(-\Delta)^{k}(g(z) y)=h+h(g(z)) & \text { in } Q, \\ \frac{\partial^{j} L}{\partial \nu^{j}}=0, j=0,1, \cdots m-1 & \text { on } \Sigma, \\ L(0)=y_{0} & \text { in } \Omega,\end{cases}
$$

satisfy

$$
\|L(z)\|_{W} \leq K\left(1+\left\|y_{0}\right\|_{L^{2}(\Omega)}+\|h\|_{L^{2}\left(0, T ; H^{-m}(\Omega)\right)}\right) \quad \forall z \in L^{2}(Q)
$$

with $K>0$ independent of $z$. Recall that $\|g(z)\|_{L^{\infty}(Q)} \leq M$ with $M$ independent of $z$. Now, let $L\left(z_{n}\right)$ be a sequence of solutions (25) with $z_{n} \in L^{2}(Q)$. We must prove that there exists a subsequence (that we rewrite as $\left.L\left(z_{n}\right)\right)$, such that

$$
\left\|L\left(z_{n} ; T\right)-L\left(z_{n+1} ; T\right)\right\|_{L^{2}(\Omega)} \rightarrow 0 \quad \text { as } n \rightarrow \infty .
$$


By a compactness result due to Aubin [1], we know that

$$
W \subset L^{2}\left(0, T ; H^{m-1}(\Omega)\right) \text { with compact imbedding. }
$$

Therefore, by (26), we can suppose that

$$
\left\|L\left(z_{n}\right)-L\left(z_{n+1}\right)\right\|_{L^{2}\left(0, T ; H^{m-1}(\Omega)\right)} \rightarrow 0 \quad \text { as } n \rightarrow \infty .
$$

Further, it is easy to prove that $L\left(z_{n}\right)-L\left(z_{n+1}\right)$ satisfies

$$
\begin{gathered}
\left\|L\left(z_{n} ; T\right)-L\left(z_{n+1} ; T\right)\right\|_{L^{2}(\Omega)}^{2} \\
\leq-\int_{0}^{T}<D^{k}\left(g\left(z_{n}\right) L\left(z_{n}\right)-g\left(z_{n+1}\right) L\left(z_{n+1}\right)\right), D^{k}\left(L\left(z_{n}\right)-L\left(z_{n+1}\right)\right)>_{H^{-k}(\Omega) \times H_{0}^{k}(\Omega)} d t \\
\quad+\int_{0}^{T}<D^{k}\left(g\left(z_{n}\right) s_{0}-g\left(z_{n+1}\right) s_{0}\right), D^{k}\left(L\left(z_{n}\right)-L\left(z_{n+1}\right)\right)>_{H^{-k}(\Omega) \times H_{0}^{k}(\Omega)} d t .
\end{gathered}
$$

Then, by (26), since $k \leq m-1$ (notice that $k=0$ if $m=1$ ),

$$
\left\|L\left(z_{n} ; T\right)-L\left(z_{n+1} ; T\right)\right\|_{L^{2}(\Omega)}^{2} \leq \widetilde{K}\left\|L\left(z_{n}\right)-L\left(z_{n+1}\right)\right\|_{L^{2}\left(0, T ; H^{m-1}(\Omega)\right)}^{2} \rightarrow 0 \quad \text { as } n \rightarrow \infty
$$

and the proof ends.

Completion of Proof of Theorem 1. From Lemma 3, we obtain that $y_{d}-L(z ; T)$ belongs to a compact set for all $z \in L^{2}(Q)$ and so, by using Propositions 3 and 1 , we obtain that

$$
\left\{\|\varphi(z)\|_{L^{1}(\mathcal{O})} v(z), z \in L^{2}(Q)\right\} \quad \text { is bounded in } L^{\infty}(Q)
$$

Thus

$$
K_{1}=\sup _{z \in L^{2}(Q)}\|\varphi(z)\|_{L^{1}(\mathcal{O})}<\infty
$$

Obviously, $u=u(z)$ satisfies

$$
\|u\|_{L^{2}(Q)} \leq K_{2} .
$$

Therefore, if we define the operator

$$
\Lambda: L^{2}(Q) \rightarrow \mathcal{P}\left(L^{2}(Q)\right)
$$

by

$$
\Lambda(z)=\{y \text { satisfies (23), (24) for some } u \text { satisfying (29) }\},
$$

we have seen that for each $z \in L^{2}(Q), \Lambda(z) \neq \emptyset$. In order to apply Kakutani's fixed point theorem, we have to check that the next properties hold:

(i) There exists a compact subset $U$ of $L^{2}(Q)$, such that for every $z \in L^{2}(Q), \Lambda(z) \subset U$.

(ii) For every $z \in L^{2}(Q), \Lambda(z)$ is a convex, compact and nonempty subset of $L^{2}(Q)$.

(iii) $\Lambda$ is upper hemicontinuous.

The proof of these properties is as follows:

(i) From Proposition 4 we know that, there exists a bounded subset $U$ of $W$ such that for every $z \in L^{2}(Q)$, $\Lambda(z) \subset U$. Now, to see that we can choose $U$ compact we shall prove that the set

$$
\mathcal{Y}=\left\{y \text { satisfying (23) for some } z \in L^{2}(Q) \text { and } u \text { satisfying (29) }\right\}
$$

is a relatively compact subset of $L^{2}(Q)$. But this is easy to prove by using that

$$
W \subset L^{2}(Q) \text { with compact imbedding }
$$

(see Lions [12] or Simon [18]). 
(ii) We have already seen that for every $z \in L^{2}(Q), \Lambda(z)$ is a nonempty subset of $L^{2}(Q)$. Further $\Lambda(z)$ is obviously convex, because $B\left(y_{d}, \varepsilon\right)$ and $\left\{u \in L^{2}(Q)\right.$ : satisfying $\left.(29)\right\}$ are convex sets. Then, we have to see that $\Lambda(z)$ is a compact subset of $L^{2}(Q)$. In (i) we have proved that $\Lambda(z) \subset U$ with $U$ compact. Let $\left(y^{n}\right)_{n}$ be a sequence of elements of $\Lambda(z)$ which converges in $L^{2}(Q)$ to $y \in U$. We have to prove that $y \in \Lambda(z)$. We know that there exist $u^{n} \in L^{2}(Q)$ satisfying (29) such that

$$
\begin{cases}y_{t}^{n}+(-\Delta)^{m} y^{n}+(-\Delta)^{k}\left(g(z) y^{n}\right)=h+h(g(z))+u^{n} \chi_{\mathcal{O}} & \text { in } Q \\ \frac{\partial^{j} y^{n}}{\partial \nu^{j}}=0, j=0,1, \cdots, m-1 & \text { on } \Sigma \\ y^{n}(0)=y_{0} & \text { in } \Omega \\ \left|y^{n}(T)-y_{d}\right|_{2} \leq \varepsilon & \end{cases}
$$

Now, by using that the controls $u^{n}$ are uniformly bounded, we deduce that $u^{n} \rightarrow u$ in the weak topology of $L^{2}(Q)$ and $u$ satisfies (29) (see Proposition III.5 of Brezis [5]). Then, using (31) and Proposition 4 we can see that $\left(y^{n}\right)_{n}$ converges to $y$ in the weak topology of $W$ (and so, by $(30)$, strongly in $L^{2}(Q)$ ). Therefore, passing to the limit in (31) we obtain

$$
\begin{cases}y_{t}+(-\Delta)^{m} y+(-\Delta)^{k}(g(z) y)=h+h(g(z))+u \chi_{\mathcal{O}} & \text { in } Q \\ \frac{\partial^{j} y}{\partial \nu^{j}}=0, j=0,1, \cdots, m-1 & \text { on } \Sigma \\ y(0)=y_{0} & \text { in } \Omega .\end{cases}
$$

Further, $v^{n}=y-y^{n}$ is solution of

$$
\begin{cases}v_{t}^{n}+(-\Delta)^{m} v^{n}+(-\Delta)^{k}\left(g(z) v^{n}\right)=\left(u-u^{n}\right) \chi_{\mathcal{O}} & \text { in } Q \\ \frac{\partial^{j} v^{n}}{\partial \nu^{j}}=0, j=0,1, \ldots, m-1 & \text { on } \Sigma \\ v^{n}(0)=0 & \text { in } \Omega\end{cases}
$$

and satisfies $v^{n} \in W$ (see Proposition 4). Further, if we "multiply" in (32) by $v^{n}$ and integrate, we obtain that

$$
\left\|v^{n}(T)\right\|_{L^{2}(\Omega)}^{2} \leq k \int_{Q}\left(u-u^{n}\right) \chi_{\mathcal{O}} v^{n} d x d t \rightarrow 0 \quad \text { as } n \rightarrow \infty .
$$

Thus $y^{n}(T)$ converges to $y(T)$ in the strong topology of $L^{2}(\Omega)$ and $\left\|y(T)-y_{d}\right\|_{2} \leq \varepsilon$. This prove that $y \in \Lambda(z)$ and concludes the proof of (ii).

(iii) We must prove that for every $z_{0} \in L^{2}(Q)$

$$
\begin{aligned}
& \limsup _{z_{n} \stackrel{L^{2}(Q)}{\longrightarrow}} z_{0} \\
& z_{0}
\end{aligned}
$$

We have seen in (ii) that $\Lambda(z)$ is a compact set, which implies that for every $n \in \mathbb{N}$ there exists $y^{n} \in \Lambda\left(z_{n}\right)$ such that

$$
\sigma\left(\Lambda\left(z_{n}\right), k\right)=\int_{Q} k(x, t) y^{n}(x, t) d x d t .
$$

Now, by (i), $\left(y^{n}\right)_{n} \subset U$ (compact set of $\left.L^{2}(Q)\right)$. Then, there exists $y \in L^{2}(Q)$ such that (after extracting a subsequence) $y^{n} \rightarrow y$ in $L^{2}(Q)$. We shall prove that $y \in \Lambda\left(z_{0}\right)$. We know that there exist $u^{n} \in L^{2}(Q)$ satisfying (29) such that

$$
\begin{cases}y_{t}^{n}+(-\Delta)^{m} y^{n}+(-\Delta)^{k}\left(g\left(z_{n}\right) y^{n}\right)=h+h\left(z_{n}\right)+u^{n} \chi_{\mathcal{O}} & \text { in } Q \\ \frac{\partial^{j} y^{n}}{\partial \nu^{j}}=0, j=0,1, \cdots, m-1 & \text { on } \Sigma \\ y^{n}(0)=y_{0} & \text { in } \Omega \\ \left|y^{n}(T)-y_{d}\right|_{2} \leq \varepsilon & \end{cases}
$$

Then there exists $u \in L^{2}(Q)$ satisfying $(29)$ such that $u^{n} \rightarrow u$ in the weak topology of $L^{2}(\mathcal{O})$. On the other hand, by using the smoothing effect of the parabolic linear equation (in a similar way to the proof of (ii)) and 
that $g \in L^{\infty}(\mathbb{R}) \cap \mathcal{C}(\mathbb{R})$, we deduce that $y$ satisfies $(23)$ and $(24)$ with $z=z_{0}$ for some $u \in L^{2}(Q)$ satisfying (29), which implies that $y \in \Lambda\left(z_{0}\right)$. Then, for every $k \in L^{2}(Q)$,

$$
\begin{gathered}
\sigma\left(\Lambda\left(z_{n}\right), k\right)=\int_{Q} k(x, t) y^{n}(x, t) d x d t \rightarrow \int_{Q} k(x, t) y(x, t) d x d t \\
\leq \sup _{\bar{y} \in \Lambda\left(z_{0}\right)} \int_{Q} k(x, t) \bar{y}(x, t) d x d t=\sigma\left(\Lambda\left(z_{0}\right), k\right),
\end{gathered}
$$

which proves that $\Lambda$ is upper hemicontinuous and conclude the proof of (iii).

Finally, if we restrict $\Lambda$ to $K=\operatorname{conv}(U)$ (the convex envelope of $U$ ), which is a compact set of $L^{2}(Q)$, it satisfies the assumptions of Kakutani's fixed point theorem. Then, $\Lambda$ has a fixed point $y \in K$. Further, by construction, there exists a control $u \in L^{2}(Q)$ satisfying (29) such that

$$
\begin{cases}y_{t}+(-\Delta)^{m} y+(-\Delta)^{k}(f(y))=h+u \chi_{\mathcal{O}} & \text { in } Q, \\ \frac{\partial^{j} y}{\partial \nu^{j}}=0, j=0,1, \cdots m-1 & \text { on } \Sigma, \\ y(0)=y_{0} & \text { in } \Omega, \\ \left|y(T)-y_{d}\right|_{2} \leq \varepsilon . & \end{cases}
$$

Therefore, $y$ is the solution that we were looking for.

Remark 2 Several generalizations seem possible. For instance, the equation of (1) could be replaced by other ones with a more general nonlinearity

$$
y_{t}+(-\Delta)^{m} y+\sum_{i=0}^{k}(-\Delta)^{i} f_{i}(y)=h+v \chi_{\omega}
$$

or a more general lower order differential operator

$$
y_{t}+(-\Delta)^{m} y+L(f(y))=h+v \chi_{\omega},
$$

with $L$ suitable linear partial differential operator of degree lower than $2 m$. The key point in those generalizations is that the unique continuation result of Lemma 1, for the associated dual problem, remains true thanks to Theorem 3.2 of Saut-Scheurer [17] and the rest of arguments of the proof of Theorem 1 apply.

\section{Non-controllability for superlinear problems.}

In this section we assume $k=0$. We shall prove a result of non-controllability for a superlinear nonlinear term with $\bar{\omega} \subset \Omega$.

Theorem 4 Let $p>1$ and let $y(t ; u)=y \in L^{2}\left(0, T ; H^{m}(\Omega)\right) \cap \mathcal{C}\left([0, T] ; L^{2}(\Omega)\right)$ a function satisfying

$$
\begin{cases}y_{t}+(-\Delta)^{m} y+|y|^{p-1} y=u \chi_{\omega} & \text { in } Q \\ y(0)=y_{0} & \text { in } \Omega\end{cases}
$$

associated to any "natural" boundary condition and with control $u \in L^{2}(Q)$. Then we can choose $y_{d} \in L^{2}(\Omega)$ and $\varepsilon>0$ such that

$$
\left\|y(T ; u)-y_{d}\right\|_{L^{2}(\Omega)}>\varepsilon \text { for any } u \in L^{2}(Q) .
$$

In order to prove Theorem 4 we introduce, previously, some auxiliar functions. Given $R>0$ we define, on $\mathbb{R}^{N}$, the functions

$$
\xi_{R}(x)=\left(R^{2}-|x|^{2}\right) / R \text { if }|x|<R, \quad \xi_{R}(x)=0 \text { if }|x| \geq R
$$

and

$$
d_{R}(x)=R-|x| \text { if }|x|<R, \quad d_{R}(x)=0 \text { if }|x| \geq R .
$$


It is clear that

$$
d_{R}(x) \leq \xi_{R}(x) \leq 2 d_{R}(x)
$$

for all $x \in \mathbb{R}^{N}$.

The following result was proved in Bernis [4].

Proposition 5 Let $s \geq 2 m$ and $R>0$. Then, for each $\varepsilon>0$ there exist a constant $C$ depending only on $N, m, s$ and $\varepsilon$ (thus independent of $R$ ) such that the following inequality holds for all $y \in H_{\text {loc }}^{m}\left(\mathbb{R}^{N}\right)$ :

$$
\left((-\Delta)^{m} y, \xi_{R}^{s} y\right)_{H_{l o c}^{-m}\left(\mathbb{R}^{N}\right) \times H_{c}^{m}\left(\mathbb{R}^{N}\right)} \geq(1-\varepsilon) \int_{\mathbb{R}^{N}} \xi_{R}^{s}\left|D^{m} y\right|^{2} d x-C \int_{\mathbb{R}^{N}} \xi_{R}^{s-2 m} y^{2} d x
$$

Remark 3 Since $s \geq 2 m, \xi_{R}^{s} \in W_{c}^{2 m, \infty}\left(\mathbb{R}^{N}\right)$. Hence $\xi_{R}^{s} \in \mathcal{C}_{c}^{m}\left(\mathbb{R}^{N}\right)$ (see e.g. Corollary IX.13 of [5]) and $\xi_{R}^{s} u \in H_{c}^{m}\left(\mathbb{R}^{N}\right)$ (see e.g. Note $I X .4$ of $\left.[5]\right)$.

Corollary 2 Let $s \geq 2 m$ and $R>0$ such that $\overline{B_{R}} \subset \Omega$. Then, for each $\varepsilon>0$ there exist a constant $C$ depending only on $N, m, s$ and $\varepsilon$ (thus independent of $R$ ) such that the following inequality holds for all $y \in H^{m}(\Omega)$ :

$$
\left((-\Delta)^{m} y, \xi_{R}^{s} y\right)_{H^{-m}(\Omega) \times H_{0}^{m}(\Omega)} \geq(1-\varepsilon) \int_{\Omega} \xi_{R}^{s}\left|D^{m} y\right|^{2} d x-C \int_{\Omega} \xi_{R}^{s-2 m} y^{2} d x .
$$

Proof. Let $\bar{y} \in H^{m}(\Omega)$ such that $\bar{y}=y$ in $\Omega$ (such $\bar{y}$ exists by standar results: see, e.g., Chapter IX of Brezis [5]). Then, by Proposition 5, the inequality holds for $\bar{y}$, but as $\overline{B_{R}} \subset \Omega$ we obtain the result.

Theorem 5 Let $p>1, r=p+1, y_{0} \in L^{2}(\Omega)$ and $u \in L^{r^{\prime}}(Q)$. Then any solution $y \in L^{r}(Q) \cap$ $L^{2}\left(0, T ; H^{m}(\Omega)\right)$ of

$$
\begin{cases}y_{t}+(-\Delta)^{m} y+|y|^{p-1} y=u & \text { in } \mathcal{D}^{\prime}(Q), \\ y(0)=y_{0} & \text { on } \Omega,\end{cases}
$$

with any "natural" boundary condition, satisfies the local estimate

$$
\begin{gathered}
\sup _{0<t<T} \int_{B_{R}} y(x, t)^{2} d x+\int_{B_{R} \times(0, T)}\left(\left|D^{m} y\right|^{2}+|y|^{r}\right) d x d t \\
\quad \leq K\left(1+\int_{B_{R_{1}} \times(0, T)}|u|^{r^{\prime}} d x d t+\int_{B_{R_{1}}} y_{0}^{2} d x\right)
\end{gathered}
$$

if $\overline{B_{R_{1}}} \subset \Omega$ and $0<R \leq R_{1}$. Moreover, the constant $K$ depends only on $N, m, p, R, R_{1}$ and $T$.

Proof of Theorem 5. We take $X_{r}=L^{r}(Q) \cap L^{2}\left(0, T ; H_{0}^{m}(\Omega)\right.$. Then the equation of (38) is satisfied in $X_{r}^{\prime}=L^{r^{\prime}}(Q)+L^{2}\left(0, T ; H^{-m}(\Omega)\right)$. Then, if $s \geq 2 m$, we can multiply (38) by $\xi_{R}^{s} y$ with the duality product $(\cdot, \cdot)_{X_{r}^{\prime} \times X_{r}}$ and we obtain

$$
\begin{gathered}
\frac{1}{2} \int_{B_{R}} \xi_{R}^{s} y(x, T)^{2} d x+\left((-\Delta)^{m} y, \xi_{R}^{s} y\right)_{L^{2}\left(0, T ; H^{-m}(\Omega)\right) \times L^{2}\left(0, T ; H_{0}^{m}(\Omega)\right)}+\left(|y|^{p-1} y, \xi_{R}^{s} y\right)_{L^{r^{\prime}}(Q) \times L^{r}(Q)} \\
=\frac{1}{2} \int_{B_{R}} \xi_{R}^{s} y_{0}(x)^{2} d x+\left(u, \xi_{R}^{s} y\right)_{L^{r^{\prime}}(Q) \times L^{r}(Q)} .
\end{gathered}
$$

Now, from Corollary 2 it follows that

$$
\begin{gathered}
\frac{1}{2} \int_{B_{R}} \xi_{R}^{s} y(x, T)^{2} d x+\int_{B_{R} \times(0, T)} \xi_{R}^{s}\left(\left|D^{m} y\right|^{2}+|y|^{r}\right) d x d t \\
\leq C \int_{B_{R}} \xi_{R}^{s} y_{0}(x)^{2} d x+C \int_{B_{R} \times(0, T)} \xi_{R}^{s-2 m} y^{2} d x d t+C \int_{B_{R} \times(0, T)} \xi_{R}^{s} u y d x d t .
\end{gathered}
$$

By (36) and (37) we can replace in $(39) \xi_{R}(x)$ by $R-|x|$ (modifying the constants). Further, writing $s-2 m=2 s / r+(s(r-2) / r)-2 m$, we can apply Hölder's or Young's inequality with exponents $q=r / 2$ and $q^{\prime}=r / r-2$ and we obtain

$$
\int_{B_{R} \times(0, T)}(R-|x|)^{s-2 m} y^{2} d x d t
$$




$$
\leq \varepsilon \int_{B_{R} \times(0, T)}(R-|x|)^{s}|y|^{r} d x d t+K(\varepsilon, q) \int_{B_{R} \times(0, T)}(R-|x|)^{s-\gamma} d x d t
$$

with

$$
K(\varepsilon, q)=\frac{1}{q^{\prime}(q \varepsilon)^{q^{\prime} / q}} \quad \text { and } \quad \gamma=\frac{2 m r}{r-2} \quad .
$$

Hence, if we choose $s>\gamma-1$, the last integral is finite and equal to $\widetilde{C} R^{s+N-\gamma}$. On the other hand, we can apply again Young's inequality and we have

$$
\int_{B_{R} \times(0, T)}(R-|x|)^{s} u y d x d t \leq \varepsilon \int_{B_{R} \times(0, T)}(R-|x|)^{s}|y|^{r} d x d t+k(\varepsilon, r) \int_{B_{R} \times(0, T)}(R-|x|)^{s}|u|^{r^{\prime}} d x d t .
$$

Thus, by changing the constants, we deduce that

$$
\begin{aligned}
& \frac{1}{2} \int_{B_{R}}(R-|x|)^{s} y(x, T)^{2} d x+\int_{B_{R} \times(0, T)}(R-|x|)^{s}\left(\left|D^{m} y\right|^{2}+|y|^{r}\right) d x d t \\
\leq & C\left(\int_{B_{R}}(R-|x|)^{s} y_{0}(x)^{2} d x+R^{s+N-\gamma}+\int_{B_{R} \times(0, T)}(R-|x|)^{s}|u|^{r^{\prime}} d x d t\right) .
\end{aligned}
$$

Finally, by replacing $R$ by $R_{1}$ and by taking into account that $R_{1}-|x| \geq R_{1}-R$ and $R_{1}-|x| \leq R_{1}$ if $|x| \leq R$ we deduce the result with

$$
K=\max \left\{C\left(\frac{R_{1}}{R_{1}-R}\right)^{s}, \frac{C R_{1}^{s+N-\gamma}}{\left(R_{1}-R\right)^{s}}\right\} .
$$

Proof of Theorem 4. It is a trivial consequence of Theorem 5 since, if $R_{1}$ satisfies $\overline{B_{R_{1}}} \subset \Omega \backslash \omega$, then

$$
\|y(u ; T)\|_{L^{2}(\Omega)}^{2} \leq K\left(1+\left\|y_{0}\right\|_{L^{2}(\Omega)}^{2}\right) \forall u \in L^{r^{\prime}}(Q) .
$$

Therefore, taking $y_{d}$ with $\left\|y_{d}\right\|_{L^{2}(\Omega)}$ large enough, we obtain (35) for $\varepsilon>0$ small enough.

Acknowledgements. We thank F. Bernis for some useful conversations.

\section{References}

[1] Aubin, J.P.: Un théorème de compacité. C. R. Acad. Sci., Paris, Serie I, T. 256, pp. 5042-5044, (1963).

[2] Aubin, J.P.: L'analyse non linéaire et ses motivations économiques. Masson. (1984).

[3] Aubin, J.P. and Ekeland, I.: Applied nonlinear Analysis. Wiley-Interscience Publication, New York, (1984).

[4] Bernis, F.: Elliptic and Parabolic Semilinear Problems without Conditions at infinity. Arch. Rat. Mech. Anal., Vol. 106, N. 3, pp. 217-241, (1989).

[5] Brézis, H.:Analyse Fonctionnelle: Théorie et applications. Masson, Paris, (1987).

[6] Cahn, J.W. and Hilliard, J.E.: Free energy of a nonuniform system. I. Interfacial free energy. J. Chem. Phys. N. 28, pp. 258-267, (1958).

[7] Díaz, J.I. and Ramos, A.M.: Positive and negative approximate controllability results for semilinear parabolic equations. To appear in Revista de la Real Academia de Ciencias Exactas, Físicas y Naturales, Madrid, (1997).

[8] Elliot, C.M. and Songmu, Z.: On the Cahn-Hilliard Equation. Arch. Rat. Mech. Anal. N. 96, pp. 339-357, (1986).

[9] Fabre, C., Puel, J.P. and Zuazua, E.: Contrôlabilité approchée de l'équation de la chaleur semilinéaire. C. R. Acad. Sci. Paris, t. 315, Série I, pp. 807-812, (1992). 
[10] Fabre, C., Puel, J.P. and Zuazua, E.: Approximate controllability of the semilinear heat equation, Proceedings of the Royal Society of Edinburgh, 125A, pp. 31-61, (1995).

[11] Haraux, A.: Nonlinear Evolution Equations. Lecture Notes in Mathematics. Springer-Verlag, Heidelberg, (1981).

[12] Lions, J.L.: Contrôle optimal de systemes gouvernés par des equations aux derivées partielles. Dunod, Paris, (1968).

[13] Lions, J.L.: Quelques méthodes de résolution des problèms aux limites non linéares. Dunod, Paris, (1969).

[14] Lions, J.L.: Remarques sur la contrôlabilité approchée. In Proceedings of Jornadas Hispano-Francesas sobre Control de Sistemas Distribuidos, Universidad de Malaga, pp. 77-88, (1990).

[15] Lions, J.L. and Magenes, E.: Problèmes aux limites non homogènes et applications, Vol. 1, Dunod, Paris, (1968).

[16] Lions, J.L. and Magenes, E.: Problèmes aux limites non homogènes et applications, Vol. 2, Dunod, Paris, (1968).

[17] Saut, J.C. and Scheurer, B.: Unique continuation for some evolution equations. J. Differential Equations, Vol. 66, N. 1, pp. 118-139, (1987).

[18] Simon, J.: Compact Sets in the Space $L^{p}(0, T ; B)$. Annali di Matematica Pura ed Applicata. Serie 4, N. 146, pp.65-96, (1987). 